\title{
Topological Construction of Spherical Analogue of a Given Euclidean Pyramid
}

\author{
Joseph Dongho*, Siméon Kemmegne Fopossi \\ Department of Mathematics and Computer Science, University of Maroua, Maroua, Cameroon \\ *Corresponding author: josephdongho@yahoo.fr
}

Received April 12, 2019; Revised May 18, 2019; Accepted June 04, 2019

\begin{abstract}
Given a regular Euclidean pyramid with square base, we use basic properties of great circle associated to it sides to prove the existence of its spherical counterpart. We also prove that its homeomorphic to its spherical counterpart.
\end{abstract}

2010 Mathematics Subject Classification: 51N10, 51N20, 51M09, 51M15, 51 H05

Keywords: pyramid, euclidean pyramid, sphere, homeomorphism

Cite This Article: Joseph Dongho, and Siméon Kemmegne Fopossi, “Topological Construction of Spherical Analogue of a Given Euclidean Pyramid.” American Journal of Applied Mathematics and Statistics, vol. 7, no. 4 (2019): 120-130. doi: 10.12691/ajams-7-4-1.

\section{Introduction}

Euclidean geometry was believed to solve all the problems that could arise in society. Despite all these we came to discover that there were some questions Euclidean geometry could not answer. When we tried to find out, it led us to non-Euclidean Geometry. Euclid, a Greek mathematician who lived in approximately 300 B.C., is credited with collecting and organizing the postulates and theorems that are studied in geometry courses. The Parallel Postulate represents one of the most controversial assumptions made by Euclid. Over the years, Euclid's Parallel Postulate has troubled mathematicians. The thought that lines may intersect at possibly infinite. In 1795, the mathematician John Playfair devised an alternative formulation of Euclid's Parallel Postulate called Playfair's Axiom. Playfair's Axiom is more useful than Euclid's Parallel Postulate, as it answers the question, 'How many lines can be drawn through a point not on a line and, at the same time, parallel to that line?' In most geometry courses, Euclid's Parallel Postulate and Playfair's Axiom are used interchangeably. A development of geometry in which the Parallel Postulate or Playfair's Axiom do not hold is known as nonEuclidean geometry. This work is part of spherical geometry and one of it aims is to propose a method which can help us to construct spherical objet from given Euclidean one. It is well know that geometry aids in our perception of the world. We can use it to de-construct our view of objects into points, lines, circles, planes and spheres. For example, some properties of triangles that we know are that it consists of three straight lines and three angles that sum to $\pi$. Can we imagine other geometries that do not give these familiar results? The Euclidean geometry that we are familiar with depends on Euclid's parallel postulate. Since it is a postulate and not a theorem, it is assumed to be true without proof. If we alter that postulate, new geometries emerge. This project explores one model of that Non-Euclidean Geometry; the spherical geometry.

In this work we solve the following problem: is there a spherical pyramid homeomorphic to a prescribed Euclidean given pyramid? We also show how our construction can be use in engineering. Notably the existence of this homeomorphism can be use in the frame work of numerical metallurgy.

The main objective of this work is to prove the existence of spherical analogue of a given regular Euclidean pyramid with square basis. We prove their existence and we also prove that there are homeomorphic to their Euclidean counterpart. In other words we have proven that the two geometric entities are topologically equivalent. By compare their volume and area we show that those entity are not isometric [2]. More precisely we show that the volume and the area of spherical counterpart is bigger than it Euclidean analogue.

More explicitly, let $S A B C D$ be a given regular Euclidean pyramid with square basis $A B C D$. We denote $\triangle S A B, \triangle S B C, \triangle S C D$ and $\triangle S D A$ the lateral faces of $S A B C D$. To construct the spherical analogue of $S A B C D$ we define and homeomorphism from $\mathrm{IR}^{3}$ to $\mathrm{IR}^{3}$ which push each sides of $S A B C D$ on its circumscribed sphere. We denote $\mathrm{S}_{S A B}, \mathrm{~S}_{S B C}, \mathrm{~S}_{S C D}$ and $\mathrm{S}_{S D A}$ respectively the image of $\triangle S A B, \triangle S B C, \triangle S C D$ and $\triangle S D A$. Then, $\mathrm{S}_{S A B} \cup \mathrm{S}_{S B C} \cup$ $\mathrm{S}_{S C D} \cup \mathrm{S}_{S D A}$ is the spherical analogue of our Euclidean pyramid. 


\section{Preliminary}

A sphere is the surface generated by the revolution of a semicircle about its diameter, which remains fixed. The term sphere is used in a two-fold signification

1. As denoting the surface.

2. The solid bounded by the surface. These correspond to the two-fold signification of the word circle in plane Geometry, namely, the circumference, and the area included within it. The centre of the generating semicircle is called the centre of the sphere. A radius of the sphere is any right line drawn from the centre to a point in the surface. A diameter of a sphere is any right line drawn through the centre, and terminated both ways by the surface.

From the definition of a spherical surface it follows at once

1. That every point in it is equally distant from the centre of the generating semi- semicircle.

2. That any point $P$ in space is outside, on, or inside the surface, according as its distance from the centre is greater than, equal to, or less than the radius.

3. That spheres having equal radius are equal.

It is well know that

Proposition 2.1. Every section of a sphere made by a plane is a circle.

From this theorem, we deduce the following properties:

i. If $R$ be the radius of the sphere, $r$ the radius of the section, $d$ the distance of the plane of section from the centre of the sphere, $r^{2}=R^{2}-d^{2}$.

ii. If $R=d, r=0$. Hence the section will reduce to a point, and the plane will touch the sphere.

iii. Two circles, whose planes are equally distant from the centre, are equal.

Between elements of sphere, one of the most useful in our work is the great circle.

Definition 2.1. Circle of the sphere whose plane passes through the centre is called a great circle, and a circle whose plane does not pass through the centre is called a small circle.

The following well know theorem will be very important in our construction.

Proposition 2.2. Only one great circle can be drawn through two points on the surface of the sphere, unless they are diametrically opposite.

Proposition 2.3. The locus of all the points of a sphere which are equidistant from two fixed points $A, B$ of the sphere is the great circle, which is perpendicular at its middle point to the arc of the great circle $A B$.

\subsection{On existence of Circumscribe Sphere to a Given Pyramid}

In this subsection we will prove the existence of circumscribe sphere to a given regular pyramid with square basis.

Let $E$ be a dimension 3 Euclidean space and $S A B C D$ five non coplanar points of $E$ such that $A B C D$ form a square. $S A B C D$ is a regular pyramid if the following triangle $\triangle S A D, \triangle S D C, \triangle S C B, \triangle S B A$ are equilateral.

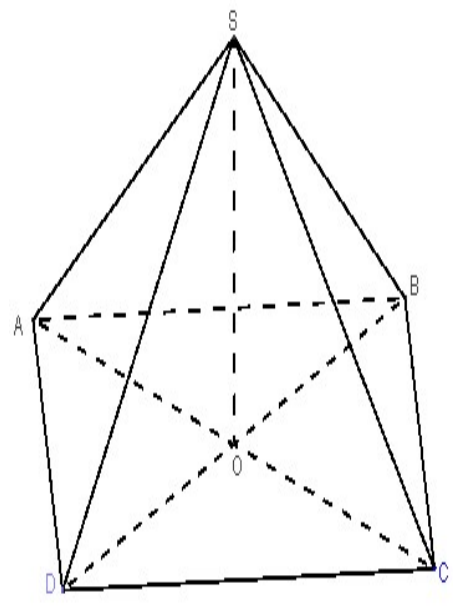

Figure 1. Regular pyramid with square

Perpendicular bisector of real line segment $[X, Y]$ of the $n \in \mathrm{IN}^{*}$ dimensional Euclidean space $E$ is the affine hyperplane $(X Y)^{\perp}$ passing through the midpoint of $[X, Y]$ and perpendicular to $(X Y)$.

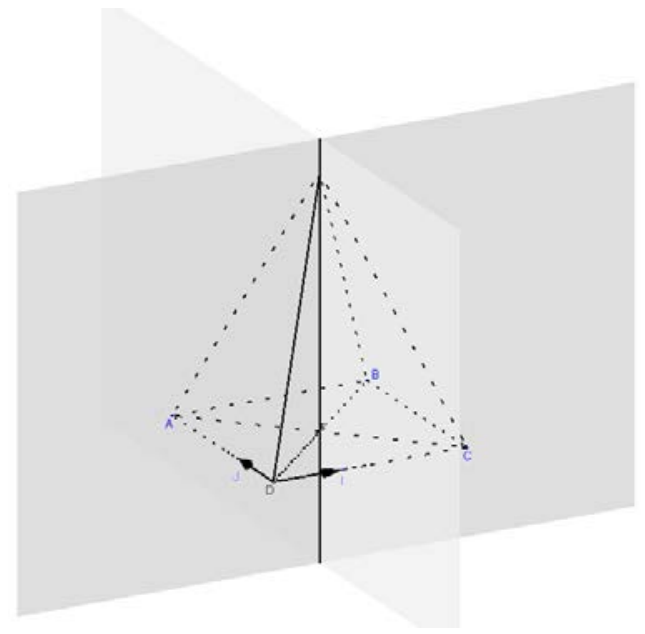

Figure 2. Perpendicular bisector

Any two great circles of the sphere bisect each other. The figure formed by the shorter arcs joining three points on the surface of a sphere, no two of which are diametrically opposite, is called a spherical triangle. The portion of a sphere included between two halves of great circles is called a lune. Two triangles, which have a common side and whose other sides belong to the same great circles, are called columnar, triangles.

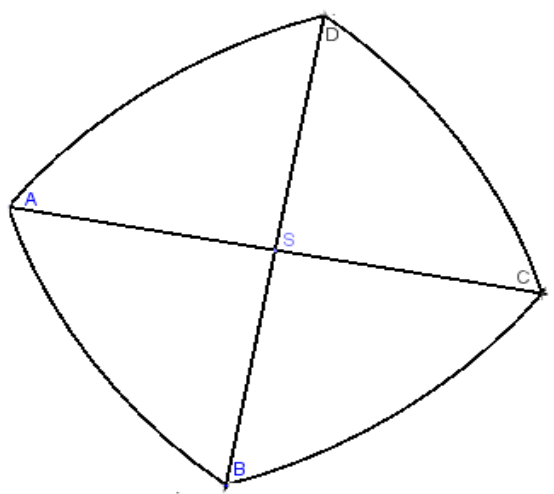

Figure 3. Circumscribe sphere to a pyramid SABCD 
Theorem 2.1. Any regular pyramid with square basis is writable in a sphere with centre in the altitude.

Proof: Let $A B C D$ be the basis of a pyramid $S A B C D$ and $[O S]$, its altitude. Then the line segments $[A B]$ and $[D C]$ have common perpendicular bisector plan $\mathrm{P}$.

As matter of the fact, $\mathrm{P}$ is perpendicular to the line segment $[A B]$ and passing through its midpoint and it is perpendicular to the line $(A B)$ since line $(A B) \|(D C)$ and $A B=D C$, then it is also according to the Thales theorem, perpendicular to $[A D]$ and it passing through its midpoint. Therefore, it is also its perpendicular bisector. Likewise $B C$ and $A D$ have the same perpendicular bisector $\mathrm{Q}$. Since point $O A=O C, O D=O B, S A=A B=S B=S C=S D$, then $(O S) \in \mathrm{P} \cap \mathrm{Q}$. Therefore $\mathrm{P} \cap \mathrm{Q}=\{(O S)\}$. Let $\mathrm{R}$ be the perpendicular bisector of $[S A]$. According to Thales theorem, $\mathrm{R}$ is the perpendicular bisector of $[S D],[S C]$ and

[SC]. Therefore, $\Omega=(O S) \cap \mathrm{R}$ is equidistance to point $A, B$, $C, D, S$. It is therefore the center of the circumscribe sphere to the pyramid $S A B C D$.

$$
\text { Denote } \mathrm{R}=(O ; \vec{i}, \vec{j}, \vec{k}) ;
$$

where $\vec{i}=\frac{\overrightarrow{O D}}{O D}, \vec{j}=\frac{\overrightarrow{O C}}{O C}, \vec{k}=\frac{\overrightarrow{O S}}{O S}$, then $(O ; \vec{i}, \vec{\jmath}, \vec{k})$.

Is direct orthonormal frame. Let $e$ be the length basis's side and $h$ the altitude of above pyramid.

Proposition 2.4. The centre of the circumscribe sphere of pyramid $S A B C D$ is $\Omega\left(0 ; 0 ; \frac{2 h^{2}-e^{2}}{4 h}\right)$ and it radius is $R=\frac{1}{2} h+\frac{e^{2}}{4 h}$.

\section{Proof:}

- Determination of the centre of circumscribe sphere. After determine coordinate of points $A, B, C, D$ and $S$ in the frame $\mathrm{R}$, we fine the component of vectors $\overrightarrow{\Omega B}, \overrightarrow{\Omega C}, \overrightarrow{\Omega D}$, $\overrightarrow{\Omega S}$ where $\Omega(x, y, z)$ is the centre of the circumscribe sphere.

We have $\Omega A=\Omega B$, if and only if $\Omega A^{2}=\Omega B^{2}$

i.e ; $x^{2}+(O C+y)^{2}+z^{2}=(O D+x)^{2}+y^{2}+z^{2}$

i.e ;

$$
O C^{2}+2 O C \cdot y=O D^{2}+2 O D \cdot x
$$

Likewise, $\Omega D=\Omega A$ if and only if $\Omega D^{2}=\Omega A^{2}$ i.e; $y^{2}$ $+(O D-x)^{2}+z^{2}=(O C+y)^{2}+x^{2}+z^{2}$

i.e;

$$
O D^{2}-2 O D \cdot x=O C^{2}+2 O C \cdot y
$$

From (1) and 2, we deduce that $2 O D^{2}=20 C^{2}+4 O C \cdot y$,

i.e; $y=\frac{O D^{2}-O C^{2}}{2 O C}$

i.e $y=0$ since $O D=O C$.

It follow from, (2) that $x=y$ because $O C=O D$. Therefore, $x=0$.

Elsewhere, $\Omega A=\Omega S$ if and only if $\Omega A^{2}=\Omega S^{2}$ i.e; $x^{2}$ $+(O C+y)^{2}+z^{2}=x^{2}+y^{2}+(O S-z)^{2}$,

i.e; $O C^{2}+z^{2}=(O S-z)^{2}$, therefore

$$
z=\frac{O S^{2}-O C^{2}}{2 O S} \text {. }
$$

Ever since, $\Omega\left(0 ; 0 ; \frac{O S^{2}-O C^{2}}{2 O S}\right)$. But $O S=h$ an according to the Pythagoreans theorem in the right triangle $A B C$, we have:

$$
O C=\frac{A C}{\sqrt{2}}=\frac{\sqrt{2}}{2} e
$$

because $\mathrm{AC}=e$.

Therefore $\Omega\left(0 ; 0 ; \frac{2 h^{2}-e^{2}}{4 h}\right)$.

- Determination of the radius of the circumscribe sphere.

By definition, we have :

$$
\mathrm{R}=\Omega A=\sqrt{O C^{2}+\left(\frac{O S^{2}-O C^{2}}{2 O S}\right)^{2}}=\frac{O S^{2}+O D^{2}}{2 O S} .
$$

Thus $R=\frac{1}{2} h+\frac{e^{2}}{4 h}$ because $O S=h$ and $O D^{2}=\frac{e^{2}}{2}$.

\subsection{On Associated Spherical Pyramid}

We suppose that $S A B C D$ is a real Euclidean pyramid. Before we construct the spherical pyramid associated to $S A B C D$, we shall first recall some analogy between the geometry of the sphere and the plane. In order to understand the analogy between plane and spherical geometry, it is necessary to observe that to right lines on the plane correspond on the sphere great circles, and the circles on the plane correspond circles on the sphere, which may be either great or small. We have proven that our Euclidean pyramid is inscribed in a sphere. Our pyramid has 8 sides and each side define with the centre $\Omega$ a unique Euclidean plan which intersect the sphere trough a great circle. It is well know that any two great circles of the sphere bisect each other. The sides of our spherical pyramid are defined by section of those great circles with the sphere.

\subsubsection{Great Circles Equations Associated to Euclidean Pyramid}

Proposition 2.5. Equations of the planes $\mathrm{P}_{\Omega,[S A]}$ and $\mathrm{P}_{\Omega,[S C]}$ passing respectively trough $\Omega, S, A$ and $\Omega, S, C$ are: $x=0$.

Proof: Let $M(x, y, z) \in \mathrm{IR}^{3} \cap \mathrm{P}_{\Omega,[S A]}$. We fine coordinates of $\overrightarrow{A M}, \overrightarrow{A S}$ and $\overrightarrow{A \Omega}$. Since those Points are coplanar, we have: $(\overrightarrow{A S} \wedge \overrightarrow{A \Omega}) \cdot \overrightarrow{A M}=0$ and then $(a-O S) O C x=0$, since $a-O S<0$, where $a=\frac{2 h^{2}-e^{2}}{4 h}$. Thus, $\mathrm{P}_{\Omega,[S A]}$ : $x=0$. Likewise, we prove that the plane passing trough $A$, $S, \Omega$ and $S, C, \Omega$ have the same equation.

Proposition 2.6. Equations of plans $\mathrm{P}_{\Omega,[S D]}$ and $\mathrm{P}_{\Omega,[S B]}$ passing through $\Omega, S, D$ and $\Omega, S, \mathrm{~B}$ is $\mathrm{y}=0$.

Proof: Let $M(x, y, z) \in \mathrm{IR}^{3} \cap \mathrm{P}_{\Omega,[S D]}$, we determine in the frame $\mathrm{R}$ the coordinates of vectors $\overrightarrow{D S}, \overrightarrow{D \Omega}$ and $\overrightarrow{D M}$. Since $D, S, \Omega$ and $M$ are coplanar, $(\overrightarrow{D S} \wedge \overrightarrow{D \Omega}) \cdot \overrightarrow{D M}=0$.

i.e $y=0$; since $a+O S \neq 0$.

Then $\mathrm{P}_{\Omega,[S D]}: y=0$. Likewise, we show that plan $\mathrm{P}_{\Omega,[S B]}$ and $\mathrm{P}_{\Omega,[S D]}$ has the same equation.

\section{Proposition 2.7.}

$2 a x+2 a y-e \sqrt{2} z+a \sqrt{2} e=0$ is the equation of the plan $\mathrm{P}_{\Omega,[A B]}$ passing through $\Omega, A$ and $B$. 
Proof: Let $M(x, y, z) \in \mathrm{IR}^{3} \cap \mathrm{P}_{\Omega,[A B] \text {, }}$

We determine the coordinates in $\mathrm{R}$ of $\overrightarrow{A M}, \overrightarrow{A B}$ and $\overrightarrow{A \Omega}$. Since $A, B, \Omega$ and $M$ are coplanar.

i.e $a . O C x+a . O D y-O D . O C z+a . O D . O C=0$. Then $a x+a y-O D z+a \cdot O D=0$, because $O D=O C$. And then

$\mathrm{P}_{\Omega,[A B]}: 2 a x+2 a y-e \sqrt{2} z+a \sqrt{2} e=0$; because $\mathrm{h}=\mathrm{OS}$ and $\mathrm{OD}=\mathrm{OC}=c \frac{\sqrt{2}}{2}$.

\section{Proposition 2.8.}

$-2 a x+2 a y-e \sqrt{2} z+a \sqrt{2} e=0$ is the equation of plan $\mathrm{P}_{\Omega,[A D]}$ passing through $\Omega, A$ and $D$.

Proof: Let $M(x, y, z) \in \mathrm{IR}^{3} \cap \mathrm{P}_{\Omega,[A D]}$. Using the coordinates in the frame $\mathrm{R}$ of $\overrightarrow{D A}, \overrightarrow{D \Omega}$ and $\overrightarrow{D M}$ and the fact that points $A, D, \Omega$ and $M$ are coplanar, $(\overrightarrow{D A} \wedge \overrightarrow{D \Omega}) \cdot \overrightarrow{D M}=0$, we deduce that Then

$$
P_{\Omega,[A D]}:-2 a x+2 a y-e \sqrt{2} z+a \sqrt{2} e=0 .
$$

\section{Proposition 2.9.}

$2 a x+2 a y+e \sqrt{2} z-a \sqrt{2} e=0$ is the equation of plan $\mathrm{P}_{\Omega,[D C]}$ passing through $\Omega, C$ and $D$

Proof: Let $M(x, y, z) \in \mathrm{IR}^{3} \cap \mathrm{P}_{\Omega,[D C]}$. After determine coordinates in the frame $\mathrm{R}$ of $\overrightarrow{C D}, \overrightarrow{C \Omega}$ and $\overrightarrow{C M}$, we use the fact that points $C, D, \Omega$ and $M$ are coplanar, to compute $(\overrightarrow{C D} \wedge \overrightarrow{C \Omega}) \cdot \overrightarrow{C M}=0$ and then obtain

$$
2 a x+2 a y+e \sqrt{2} z-a \sqrt{2} e=0 .
$$

\section{Proposition 2.10.}

$-2 a x+2 a y+e \sqrt{2} z-a \sqrt{2} e=0$ is the equation of plan $\mathrm{P}_{\Omega,[B C]}$ passing through point $\Omega, C$ and $B$.

Proof: Let $M(x, y, z) \in \mathrm{IR}^{3} \cap \mathrm{P}_{\Omega,[B C]}$. Using coordinates in the frame $\mathrm{R}$ of $\overrightarrow{B M}, \overrightarrow{B \Omega}$ and $\overrightarrow{B C}$, and the fact that $B, A, \Omega$ and $M$ are coplanar, i.e $(\overrightarrow{B C} \wedge \overrightarrow{B \Omega}) \cdot \overrightarrow{B M}=0$, we obtain:

$$
\begin{gathered}
-a O C x+a O D y+O D^{2} z-a O D^{2}=0 \\
2 a x+2 a y+e \sqrt{2} z-a \sqrt{2} e=0 .
\end{gathered}
$$

Therefore

$$
P_{S,[B C]}:-2 a x+2 a y+e \sqrt{2} z-a \sqrt{2} e=0 .
$$

Proposition 2.11. The equation of the great circle $\mathrm{P}_{A B C}$ passing through points $B, A$ and $C$ is: $z=0$ in R.

Proof: If $M(x, y, z) \in \mathrm{IR}^{3} \cap \mathrm{P}_{A B C}$, then using coordinate of $\overrightarrow{A M}, \overrightarrow{A B}$ and $\overrightarrow{A C}$ and the fact that points $A, B, C$ and $M$ are coplanar,

$$
\text { i.e; }(\overrightarrow{A B} \wedge \overrightarrow{A C}) \cdot \overrightarrow{A M}=0 \text {. That }-2 \mathrm{OD}^{2} \mathrm{z}=0 \text {. }
$$

Therefore, PABC: $z=0$.

We recall that; when two arcs of circles intersect, the angle of the tangents at their points of intersection is called the angle of the arcs. It is also well know that; the angle of intersection of two great circles is equal to the inclination of their planes. We conclude that, the angle between two great circles is equal to the inclination of their planes. Therefore, we have a method to fine angle between two spherical line segments. Since the lateral surfaces of our spherical pyramid are deformed of their Euclidean counterpart. We need to define the spherical triangle. The special case of antipodal triangle we play a major role in this work. We recall its definition.
Definition 2.2. Two triangles, whose corresponding vertices are diametrically opposite, are called antipodal triangle.

After defined spherical angle, we need to give the expression of some their trigonometric functions.

Proposition 2.12. Let $\theta$, be an oriented angle between plans $(A O B)$ and $(A \Omega B)$. Then:

$$
\left\{\begin{array}{l}
\cos \theta=\frac{2 e h \sqrt{4 h^{4}+e^{4}}}{4 h^{4}+e^{4}} \\
\sin \theta=\frac{\left(2 h^{2}-e^{2}\right) \sqrt{4 h^{4}+e^{4}}}{4 h^{4}+e^{4}}
\end{array}\right.
$$

Where $e$ is the basis side length and $h$ the altitude of pyramid $S A B C D$ of Figure 1.

Proof: $\theta$ is equal to angle between perpendicular the plans $\overline{(A O B)}$ and $(A \Omega B)$ Equation of the perpendicular bisector to $[A B]$ is $y=x$.

Indeed, let $I$ be the midpoint of $[A B]$, then $I\left(-\frac{O D}{2} ;-\frac{O D}{2} ; 0\right)$. Since $\overrightarrow{A B}$ is orthogonal to $\overrightarrow{I M}$, if $M \in \mathrm{P}_{\text {med }}[A B]$, then $\overrightarrow{A B} \cdot \overrightarrow{I M}=0$,

$$
\text { i.e; }\left(\mathrm{x}+\frac{O D}{2}\right)-\left(\mathrm{y}+\frac{O D}{2}\right)=0 \text {. Thus } \mathrm{y}=0 \text {. }
$$

Therefore $\mathrm{P}_{\text {med }}[A B]: y=x$.

Denote $\Delta_{1}=\mathrm{P}_{\text {med }}[A B] \cap \mathrm{P}_{O,[A B]}$ and $\Delta_{2}=\mathrm{P}_{\text {med }}[A B] \cap \mathrm{P}_{\Omega,[A B]}$.

The equation of plan $\mathrm{P}_{0,[A B]}$ is: $z=0$ and we have $\mathrm{P}_{\Omega,[A B]}$ : $a x+a y-O D z+a O D=0$ where

$$
\begin{gathered}
a=\frac{2 h^{2}-e^{2}}{4 h}, \Delta_{1}:\left\{\begin{array}{l}
y=x \\
z=0
\end{array}\right. \\
\Delta_{2}:\left\{\begin{array}{c}
y=x \\
a x+a y-O D z+a O D=0
\end{array}\right.
\end{gathered}
$$

And then $\overrightarrow{u_{1}}\left(\begin{array}{l}2 \\ 2 \\ 0\end{array}\right)$ is direction of $\overrightarrow{\Delta_{1}}$ and $\overrightarrow{u_{2}}\left(\begin{array}{c}2 O D \\ 2 O D \\ 4 a\end{array}\right)$ that of $\overrightarrow{\Delta_{2}}$. Yet $\overrightarrow{u_{1}} \cdot \overrightarrow{u_{2}}=u_{1} u_{2} \cos \theta$ and $\left\|\overrightarrow{u_{1}} \wedge \overrightarrow{u_{2}}\right\|=u_{1} u_{2}|\sin \theta|$ where $u_{1}=\sqrt{4+4}=2 \sqrt{2}$ and

$$
u_{2}=\sqrt{4 O D^{2}+4 O D^{2}+16 a^{2}}=\frac{2 \sqrt{O S^{4}+O D^{4}}}{O S} .
$$

Somewhere else, $\overrightarrow{u_{1}} \cdot \overrightarrow{u_{2}}=4 \mathrm{OD}+4 \mathrm{OD}=8 \mathrm{OD}$.

$$
\begin{aligned}
\cos \theta & =\frac{\overrightarrow{u_{1}} \cdot \overrightarrow{u_{2}}}{u_{1} u_{2}} \\
& =\frac{2 O D O S}{\sqrt{2 O S^{4}+2 O D^{4}}} \\
& =\frac{2 e h \sqrt{4 h^{4}+e^{4}}}{4 h^{4}+e^{4}} .
\end{aligned}
$$

Besides, $\overrightarrow{u_{1}} \wedge \overrightarrow{u_{2}}=\left(\begin{array}{c}8 a \\ -8 a \\ 4 O D-4 O D\end{array}\right)=\left(\begin{array}{c}8 a \\ -8 a \\ 0\end{array}\right)$. Therefore, 


$$
\begin{aligned}
|\sin \theta| & =\frac{8|a| \sqrt{2}}{2 \sqrt{2} \times \frac{2 \sqrt{O S^{4}+O D^{4}}}{O S}} \\
& =\frac{O D^{2}-O S^{2}}{\sqrt{O S^{4}+O D^{4}}} .
\end{aligned}
$$

Since $\theta$ is obtuse and negatif, we have $\cos \theta>0$ and $\sin \theta<0$,

$$
\begin{aligned}
\sin \theta & =\frac{\left(O S^{2}-O D^{2}\right) \sqrt{O S^{4}+O D^{4}}}{O S^{4}+O D^{4}} \\
& =\frac{\left(2 h^{2}-e^{2}\right) \sqrt{4 h^{4}+e^{4}}}{4 h^{4}+e^{4}} .
\end{aligned}
$$

Because 0D $>$ OS.

Proposition 2.13. There exist an Euclidean rotation which transform plan $\mathrm{P}_{0,[A B]}$ to plan $\mathrm{P} \Omega,[A B]$.

Proof: The unit direct vector of line (AB) is $\vec{u}\left(\begin{array}{c}\frac{\sqrt{2}}{2} \\ -\frac{\sqrt{2}}{2} \\ 0\end{array}\right)$.

According to Rodrigues formulas the matrix of the rotation in the frame $\mathrm{R}$ is

$$
\begin{aligned}
R_{\vec{u} \theta,[A B]}= & \cos \theta\left(\begin{array}{ccc}
1 & 0 & 0 \\
0 & 1 & 0 \\
0 & 0 & 1
\end{array}\right) \\
& +(1-\cos \theta)\left(\begin{array}{ccc}
a_{1}^{2} & a_{1} a_{2} & a_{1} a_{3} \\
a_{1} a_{2} & a_{2}^{2} & a_{2} a_{3} \\
a_{1} a_{3} & a_{2} a_{3} & a_{3}^{2}
\end{array}\right) \\
& +\sin \theta\left(\begin{array}{ccc}
0 & -a_{3} & a_{2} \\
a_{3} & 0 & -a_{1} \\
-a_{2} & a_{1} & 0
\end{array}\right) .
\end{aligned}
$$

Where $a_{1}, a_{2}$ and $a_{3}$ are coordinates of $\vec{u}$ in R. Now

$$
\begin{gathered}
\cos \theta\left(\begin{array}{lll}
1 & 0 & 0 \\
0 & 1 & 0 \\
0 & 0 & 1
\end{array}\right)=\left(\begin{array}{ccc}
\cos \theta & 0 & 0 \\
0 & \cos \theta & 0 \\
0 & 0 & \cos \theta
\end{array}\right) \\
(1-\cos \theta)\left(\begin{array}{ccc}
a_{1}^{2} & a_{1} a_{2} & a_{1} a_{3} \\
a_{1} a_{2} & a_{2}^{2} & a_{2} a_{3} \\
a_{1} a_{3} & a_{2} a_{3} & a_{3}^{2}
\end{array}\right) \\
=\left(\begin{array}{ccc}
\frac{1}{2}-\frac{\cos \theta}{2} & -\frac{1}{2}+\frac{\cos \theta}{2} & 0 \\
-\frac{1}{2}+\frac{\cos \theta}{2} & \frac{1}{2}-\frac{\cos \theta}{2} & 0 \\
0 & 0 & 0
\end{array}\right)
\end{gathered}
$$

$$
\begin{aligned}
& \sin \theta\left(\begin{array}{ccc}
0 & -a_{3} & a_{2} \\
a_{3} & 0 & -a_{1} \\
-a_{2} & a_{1} & 0
\end{array}\right) \\
& =\left(\begin{array}{ccc}
0 & 0 & \frac{-\sqrt{2} \sin \theta}{2} \\
0 & 0 & \frac{-\sqrt{2} \sin \theta}{2} \\
\frac{\sqrt{2} \sin \theta}{2} & \frac{\sqrt{2} \sin \theta}{2} & 0
\end{array}\right) .
\end{aligned}
$$

Consequently,

$$
R_{\vec{u},[A B]}=\left(\begin{array}{ccc}
\frac{1+\cos \theta}{2} & \frac{-1+\cos \theta}{2} & \frac{-\sqrt{2} \sin \theta}{2} \\
\frac{-1+\cos \theta}{2} & \frac{1+\cos \theta}{2} & \frac{-\sqrt{2} \sin \theta}{2} \\
\frac{\sqrt{2} \sin \theta}{2} & \frac{\sqrt{2} \sin \theta}{2} & \cos \theta
\end{array}\right) .
$$

Proposition 2.14. The image of frame $\mathrm{R}=(0 ; \vec{i}, \vec{j}, \vec{k})$ by $R_{\overrightarrow{u \theta},[A B]}$ is the directed frame $\mathrm{R}^{\prime}=\left(\mathrm{O}^{\prime} ; \vec{i}^{\prime}, \overrightarrow{j^{\prime}}, \overrightarrow{k^{\prime}}\right)$. Where $O^{\prime}\left(\begin{array}{l}\frac{-1+\cos \theta}{2} O D \\ 2\end{array}\right), \quad \overrightarrow{i^{\prime}}\left(\begin{array}{c}\frac{1+\cos \theta}{2} \\ \frac{-1+\cos \theta}{2} \\ \frac{\sqrt{2} \sin \theta}{2} O D\end{array}\right), \quad \overrightarrow{j^{\prime}}\left(\begin{array}{c}\frac{-1+\cos \theta}{2} \\ \frac{1+\cos \theta}{2} \sin \theta \\ 2\end{array}\right)$, and $\overrightarrow{k^{\prime}}\left(\begin{array}{c}-\frac{\sqrt{2} \sin \theta}{2} \\ \frac{\sqrt{2} \sin \theta}{2} \\ \cos \theta\end{array}\right)$, are coordinates of $O^{\prime}, \overrightarrow{i^{\prime}}, j^{\prime}$ and $\overrightarrow{k^{\prime}}$ in the frame $R$, and the basis $(\vec{i}, \vec{j}, \vec{k})$.

Proof: We have:

$$
\overrightarrow{i^{\prime}}=R_{\vec{u},[A B]} \vec{i}=\left(\begin{array}{c}
\frac{1+\cos \theta}{2} \\
\frac{-1+\cos \theta}{2} \\
\frac{\sqrt{2} \sin \theta}{2}
\end{array}\right) \text { since } \vec{i}\left(\begin{array}{l}
1 \\
0 \\
0
\end{array}\right) .
$$

Likewise,

$$
\overrightarrow{j^{\prime}}=R_{\overrightarrow{u \theta},[A B]} \vec{j}=\left(\begin{array}{l}
\frac{-1+\cos \theta}{2} \\
\frac{1+\cos \theta}{2} \\
\frac{\sqrt{2} \sin \theta}{2}
\end{array}\right) \text { since } \vec{j}\left(\begin{array}{l}
0 \\
1 \\
0
\end{array}\right) .
$$

Then, 


$$
\overrightarrow{k^{\prime}}=R_{\vec{u},[A B]} \vec{k}=\left(\begin{array}{c}
-\frac{\sqrt{2} \sin \theta}{2} \\
\frac{\sqrt{2} \sin \theta}{2} \\
\cos \theta
\end{array}\right) \text { sincek }\left(\begin{array}{l}
0 \\
0 \\
1
\end{array}\right) \text {. }
$$

We shall determine coordinates of $O^{\prime}$ to conclude.

Let $f_{\overrightarrow{u \theta},[A B]}$, be the affine rotation to the vectorial rotation above, then $f_{\overrightarrow{u \theta},[A B]}(\mathrm{A})=\mathrm{A}$; i.e;

$$
\left\{\begin{array}{c}
0=\frac{-1+\cos \theta}{2} O D+d_{1} \\
-O D=\frac{-1+\cos \theta}{2} O D+d_{2} \\
0=\frac{\sqrt{2} \sin \theta}{2} O D+d_{3}
\end{array}\right.
$$

Where

$$
\begin{aligned}
& \left(d_{1} ; d_{2} ; d_{3}\right)=f_{\vec{u} \theta,[A B]}(O) \\
& =O^{\prime}\left(\frac{-1+\cos \theta}{2} O D, \frac{-1+\cos \theta}{2} O D, \frac{\sqrt{2} \sin \theta}{2} O D\right) .
\end{aligned}
$$

$\mathrm{R}^{\prime}$ is the direct orthogonal frame image of the frame $\mathrm{R}$ by an isometry.

\section{Topological Relation between the Two Pyramids}

In this section, we suppose that we are in three dimensional Euclidean real space and we construct a homeomorphism between Euclidean pyramid and his spherical counterpart.

\subsection{Cartesian Equation of $S A D$}

\section{Lemma 3.1.}

$$
\mathcal{P}_{S,[A D]}: h x-h y+c \frac{\sqrt{2}}{2} z-c \frac{\sqrt{2}}{2} h=0
$$

is the equation of the plan $\mathrm{P}_{S,[A D]}$ passing through points $S$, $A$ and $D$ in $\mathrm{R}$.

Proof: Fine coordinates of points $A, D$ and $S$; and for any $M(x ; y ; z) \in \mathrm{IR}^{3} \cap \mathcal{P}_{S,[A D]}$ determine the coordinates of vectors $\overrightarrow{S A}, \overrightarrow{S D}$ and $\overrightarrow{S M}$ in the frame $\mathrm{R}$ and compute $(\overrightarrow{S A} \wedge \overrightarrow{S D}) \cdot \overrightarrow{S M}=0$. Therefore OSx $-\mathrm{OSy}+\mathrm{ODz}-\mathrm{ODOS}=$ 0 then $\mathcal{P}_{S,[A D]}: h x-h y+c \frac{\sqrt{2}}{2} z-c \frac{\sqrt{2}}{2} h=0$. . Because OS $=\mathrm{h}$ and $\mathrm{OD}=\mathrm{c} \frac{\sqrt{2}}{2}$.

\section{Lemma 3.2.}

$(S D):\left\{\begin{array}{l}x=\frac{-\sqrt{2} c}{2 h} z+\frac{\sqrt{2}}{2} c \\ y=0\end{array} ;(S A):\left\{\begin{array}{l}y=\frac{\sqrt{2} c}{2 h} z-\frac{\sqrt{2} c}{2} \\ x=0\end{array}\right.\right.$ and
$(A D):\left\{\begin{array}{l}x=y+O D \\ z=0\end{array}\right.$ are equations of Euclidean lines $(S D)$, $(S A)$ and $(A D)$ respectively.

Proof: In the frame $R$ we have:

- $M(x ; y ; z) \in(S D)$ if and only if $\overrightarrow{S M} \wedge \overrightarrow{S D}=\overrightarrow{0}$. i.e;

$$
\left\{\begin{array}{c}
-O S y=0 \\
O S x+O D(z-O S)=0 \\
-O D y=0
\end{array}\right.
$$

Thus

$(S D):\left\{M(x, y, z) \in I R^{3} / x=-\frac{\sqrt{2} c}{2 h} z+\frac{\sqrt{2}}{2} c ; y=0\right\}$

- In the same manner, we prove that

$$
(S A):\left\{M(x, y, z) \in I R^{3} / x=0 ; y=\frac{\sqrt{2} c}{2 h} z-\frac{\sqrt{2}}{2} c\right\}
$$

and

$$
(A D):\left\{M(x, y, z) \in I R^{3} / x=y+\frac{\sqrt{2}}{2} c ; z=0\right\} .
$$

Proposition 3.1. Let $\Delta_{S A D}$, be the region bounded by the Euclidean triangle $S A D$.

Then

$$
\Delta_{S A D}=\left\{\begin{aligned}
& h x-h y+c \frac{\sqrt{2}}{2} z-c \frac{\sqrt{2}}{2} h=0 \\
& M(x, y, z) \in I R^{3}: {\left[\begin{array}{l}
c \frac{\sqrt{2}}{2 h} z-c \frac{\sqrt{2}}{2} \\
\leq x+y \\
\leq-c \frac{\sqrt{2}}{2} z+c \frac{\sqrt{2}}{2}
\end{array}\right] } \\
& 0 \leq z \leq h
\end{aligned}\right\}
$$

is the equation of $\Delta_{S A D}$ in the frame $\mathrm{R}$.

\subsection{Expression of Associates Spherical Pyramid}

\section{Proposition 3.2.}

$$
\begin{aligned}
& \Delta_{S A D} \\
& =\left\{M(X, Y, Z) \in I R^{3}:\left[\begin{array}{l}
X \frac{f}{2}-Y \frac{f}{2}+Z \frac{\sqrt{2}}{2} g-c \frac{\sqrt{2}}{2} t=0 \\
{[|X+Y| f} \\
\left.\leq-c Z \sqrt{2}+c t p \sqrt{2}+c t q \frac{\sqrt{2}}{2 h} i\right] \\
-a p+\frac{h-i a}{2 h} q \leq Z \leq t p+\frac{i t}{2 h} q
\end{array}\right\}\right.
\end{aligned}
$$

Where: $\mathrm{f}=2 \mathrm{hp}-\mathrm{cq}$; $\mathrm{g}=2 \mathrm{hq}+\mathrm{cp} ; \mathrm{i}=1-\mathrm{c}$; and $\mathrm{t}=\mathrm{h}-\mathrm{a}$ is the expression of $\Delta_{S A D}$ in the frame R'.

proof: The first equation is just equation of plan $\mathrm{P}_{S,[A D]}$ in the frame R'. It remain the associate constraint on $X, Y$ and $Z$. By replacing expressions of $x, y$ and $Z$ as functions of $X$, $Y$ and $Z$ in the proof of lemma 3.1 we obtain: 


$$
\begin{aligned}
& \frac{c \sqrt{2}}{2 h}\left(a-\frac{\sqrt{2} \sin \theta}{2} X+\frac{\sqrt{2} \sin \theta}{2} Y+Z \cos \theta\right)-\frac{c \sqrt{2}}{2} \\
\leq & \frac{1-\cos \theta}{2} X+\frac{1+\cos \theta}{2} Y-\frac{\sqrt{2} \sin \theta}{2} Z \leq 0 \\
0 & \leq \frac{1+\cos \theta}{2} X+\frac{1-\cos \theta}{2} Y+\frac{\sqrt{2} \sin \theta}{2} Z \\
\leq & \frac{-c \sqrt{2}}{2 h}\left(a-\frac{\sqrt{2} \sin \theta}{2} X+\frac{\sqrt{2} \sin \theta}{2} Y+Z \cos \theta\right)+\frac{c \sqrt{2}}{2} \\
& 0 \leq a-\frac{\sqrt{2} \sin \theta}{2} X+\frac{\sqrt{2} \sin \theta}{2} Y+Z \cos \theta \leq h .
\end{aligned}
$$

Therefore,

$$
Y-X=\frac{\sqrt{2}\left(h \sin \theta+\frac{c}{2} \cos \theta\right)}{h \cos \theta-\frac{c}{2} \sin \theta} Z+\frac{\frac{c \sqrt{2}}{2}(a-h)}{h \cos \theta-\frac{c}{2} \sin \theta} .
$$

From in equations $(6)+(7)$ we deduce that:

$$
\begin{aligned}
& |X+Y| \leq-\frac{c \sqrt{2}}{2 h}\left(\begin{array}{l}
a-\frac{\sqrt{2} \sin \theta}{2} X \\
+\frac{\sqrt{2} \sin \theta}{2} Y+Z \cos \theta
\end{array}\right)+\frac{c \sqrt{2}}{2} \\
& \leq-\frac{c \sqrt{2}}{2 h}\left(\begin{array}{c}
a+\frac{2 h}{2 h \cos \theta-\operatorname{csin} \theta} Z \\
+\frac{a-h}{2 h \cos \theta-\sin \theta} \sin \theta
\end{array}\right)+\frac{c \sqrt{2}}{2} .
\end{aligned}
$$

According to (9),

Thus

$$
\begin{aligned}
& (2 h \cos \theta-\sin \theta)|X+Y| \\
\leq & -c \sqrt{2} Z+\frac{(h-a) c \sqrt{2}}{2 h} \sin \theta \\
& +\frac{c \sqrt{2}}{2}\left(1-\frac{a}{h}\right)(2 h \cos \theta-\sin \theta) \\
\leq & -c \sqrt{2} Z+h c \sqrt{2}\left(1-\frac{a}{h}\right) \cos \theta+\frac{c \sqrt{2}}{2 h}(h-a)(1-c) \sin \theta .
\end{aligned}
$$

Combine (9) and (8), we obtain:

$$
0 \leq a+\frac{\sqrt{2} \sin \theta}{2}\left[\begin{array}{l}
\frac{\sqrt{2}\left(h \sin \theta+\frac{c}{2} \cos \theta\right)}{h \cos \theta-\frac{c}{2} \sin \theta} \\
+\frac{c \sqrt{2}(a-h)}{2 h \cos \theta-c \sin \theta}
\end{array}\right]+Z \cos \theta \leq h
$$

i.e;

$$
\begin{aligned}
0 & \leq a(2 h \cos \theta-c \sin \theta)+2 h Z+(a-h) \sin \theta \\
& \leq h(2 h \cos \theta-c \sin \theta) .
\end{aligned}
$$

Then

$$
\begin{aligned}
& -a \cos \theta+\frac{(h-a+a c)}{2 h} \sin \theta \leq Z \\
& \leq(h-a) \cos \theta+\frac{h(1-c)+a(c-1)}{2 h} \sin \theta .
\end{aligned}
$$

This completes the proof.

Equation of circumscribe sphere in the frame $\mathrm{R}^{\prime}$ is: $X^{2}+Y^{2}+Z^{2}=R^{2}$.

Let $M \in \mathrm{IR}^{3}-\{\Omega\}$, consider the half line [ $\left.\Omega M\right]$, and denote $M^{\prime}$ a point of $[\Omega M) \cap \mathrm{S}(\Omega, R)$. Existence of $M^{\prime}$ is due to the fact that for all $M \in \mathrm{IR}^{3}-\{\Omega\}, \Omega$ is inside the domain bounded by the sphere. Consider the mapping from $\operatorname{IR}^{3}-\{\Omega\}$ to the sphere, which associate a point $M$ to point $M^{\prime}$ as is showing in the figure. This map is well defined by construction.

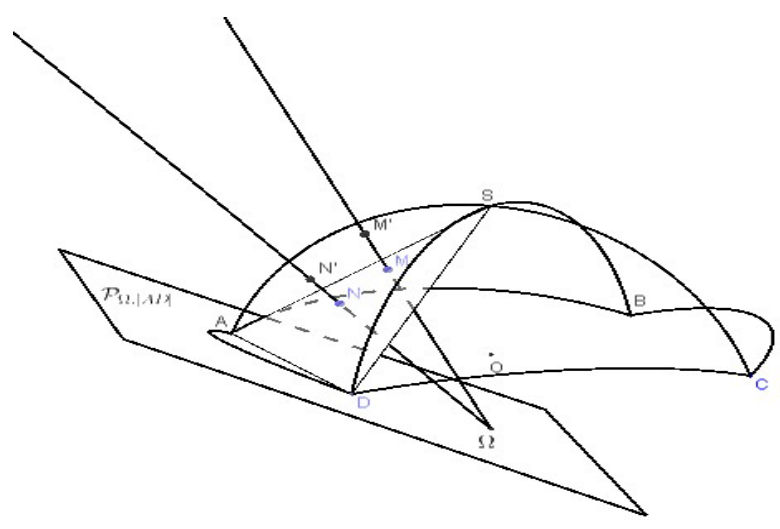

Figure 4. Image of point $M$

Lemma 3.3. In frame $\mathrm{R}$; for any $M(X ; Y ; Z) \in \Delta_{S A D}$, we have: $Z \geq 0$.

Proof: It is enough to prove that $A$ or $D$, verify the inequality.

$\mathrm{R}^{\prime}$ is image of $\mathrm{R}$ by a rotation, and the vector $\vec{k}$ is up oriented Therefore, the image $\overrightarrow{k^{\prime}}$ is also up directed. Furthermore, points $A$ and $D$ are in the plan generated by the basis $\left(\overrightarrow{i^{\prime}}, \overrightarrow{j^{\prime}}\right)$, then the third component of $A$ or $D$ is positive. Proposition 3.3. $\Delta_{S A D}$ and $S_{S A D}$ are close subset in of $\mathrm{IR}^{3}$.

Proof: It is enough to prove that their complementary $\triangle_{S A D}^{c}$ and $S_{S A D}^{c}$ of $\Delta_{S A D}$ and $S_{S A D}$ are open subset of $\mathrm{IR}^{3}$. Since $\Delta_{S A D} \subset \mathrm{IR}^{3}$, then $\Delta_{S A D}^{c} \neq \emptyset$. Let $M_{0} \in \Delta_{S A D}^{c}$. Denote $r$ $=d\left(M_{0}, \Delta_{S A D}\right)$, then $r>0$ because $M_{0} \notin \Delta_{S A D}$. Therefore $\mathrm{B}\left(M_{0}, r\right) \subset \Delta^{c} S A D$; this imply that $\Delta^{c}{ }_{S A D}$ is open, therefore $\Delta_{S A D}$ is close subset of $\mathrm{IR}^{3}$.

In the same manner, we prove that $S_{S A D}$ is close subset of $\mathrm{IR}^{3}$.

Proposition 3.4. The map:

$$
\text { With: }\left\{\begin{array}{l}
f_{1}: \Delta_{S A D} \rightarrow S_{S A D} \\
X^{\prime}=\frac{R}{\sqrt{X^{2}+Y^{2}+Z^{2}}} X \\
Y^{\prime}=\frac{R, Z) \rightarrow M^{\prime}\left(X^{\prime}, Y^{\prime}, Z^{\prime}\right)}{\sqrt{X^{2}+Y^{2}+Z^{2}}} Y \text { is an homeomorphism. } \\
Z^{\prime}=\frac{R}{\sqrt{X^{2}+Y^{2}+Z^{2}}} Z
\end{array}\right.
$$


Proof: We will prove that $f_{1}$ and its inverse $f_{1}^{-1}$ are continuous $f_{1}$ is well defined, because it is a restriction of well-defined map. The image of the Euclidean line segments $[S A],[S D]$, and $[A D]$ are spherical line segments.

- Let us prove that $f_{1}$ is bijective.

\section{a) $\mathbf{f}_{1}$ is injective.}

Let $M(X ; Y ; Z), M_{0}\left(X_{0} ; Y_{0} ; Z_{0}\right) \in \Delta_{S A D}$ such that $f_{1}(M)=$ $f_{1}\left(M_{0}\right)$. Let us prove that $M=M_{0} \cdot f_{1}(M)=f_{1}\left(M_{0}\right)$ imply that

$$
\left\{\begin{array}{l}
\frac{R}{\sqrt{X^{2}+Y^{2}+Z^{2}}} X=\frac{R}{\sqrt{X_{0}^{2}+Y_{0}^{2}+Z_{0}^{2}}} X_{0}(E 1) \\
\frac{R}{\sqrt{X^{2}+Y^{2}+Z^{2}}} Y=\frac{R}{\sqrt{X_{0}^{2}+Y_{0}^{2}+Z_{0}^{2}}} Y_{0} \quad(E 2) \\
\frac{R}{\sqrt{X^{2}+Y^{2}+Z^{2}}} Z=\frac{R}{\sqrt{X_{0}^{2}+Y_{0}^{2}+Z_{0}^{2}}} Z_{0} \quad(E 3)
\end{array}\right.
$$

To prove that $M=M_{0}$, it is enough to justify that: $\sqrt{X^{2}+Y^{2}+Z^{2}}=\sqrt{X_{0}^{2}+Y_{0}^{2}+Z_{0}^{2}}$.

By subtracting the members of $(E 1)$ and $(E 2)$, we obtain:

$$
\frac{X-Y}{\sqrt{X^{2}+Y^{2}+Z^{2}}}=\frac{X_{0}-Y_{0}}{\sqrt{X_{0}^{2}+Y_{0}^{2}+Z_{0}^{2}}} .
$$

Extracting $X-Y$ in (E4) and replacing it in (3), we obtain:

$$
\begin{aligned}
& \left(h p-\frac{c q}{2}\right)\left(\frac{\sqrt{X^{2}+Y^{2}+Z^{2}}}{\sqrt{X_{0}^{2}+Y_{0}^{2}+Z_{0}^{2}}}\right) \\
& +\sqrt{2}\left(h q+\frac{c p}{2}\right) Z \frac{c \sqrt{2}}{2}(a-h)=0 .
\end{aligned}
$$

Since $M_{0} \in \Delta_{S A D}$, then:

$$
\begin{aligned}
& \left(h p-\frac{c q}{2}\right)\left(\frac{\sqrt{X^{2}+Y^{2}+Z^{2}}}{\sqrt{X_{0}^{2}+Y_{0}^{2}+Z_{0}^{2}}}\right) \\
& \times\left[\frac{-\sqrt{2}\left(h q+\frac{c p}{2}\right) Z_{0}-\frac{c \sqrt{2}}{2}(a-h)}{h p-\frac{c q}{2}}\right] \\
& +\sqrt{2}\left(h q+\frac{c p}{2}\right) Z+\frac{c \sqrt{2}}{2}(a-h)=0 .
\end{aligned}
$$

But it follows from (3), that:

$$
Z=\frac{\sqrt{X^{2}+Y^{2}+Z^{2}}}{\sqrt{X_{0}^{2}+Y_{0}^{2}+Z_{0}^{2}}} Z_{0} .
$$

Replacing (E6) in (E5), we obtain:

$$
\begin{aligned}
& -f \frac{\sqrt{2}}{2}\left(\frac{\sqrt{X^{2}+Y^{2}+Z^{2}}}{\sqrt{X_{0}^{2}+Y_{0}^{2}+Z_{0}^{2}}}\right) Z_{0}-\frac{c \sqrt{2}}{2} t\left(\frac{\sqrt{X^{2}+Y^{2}+Z^{2}}}{\sqrt{X_{0}^{2}+Y_{0}^{2}+Z_{0}^{2}}}\right) \\
& +f \frac{\sqrt{2}}{2}\left(\frac{\sqrt{X^{2}+Y^{2}+Z^{2}}}{\sqrt{X_{0}^{2}+Y_{0}^{2}+Z_{0}^{2}}}\right) Z_{0}+\frac{c \sqrt{2}}{2} t=0 .
\end{aligned}
$$

With: $f=2 h q+c p$ and $t=a-h$.

This implies that:

$$
\frac{c \sqrt{2}}{2}(a-h)\left(1-\frac{\sqrt{X^{2}+Y^{2}+Z^{2}}}{\sqrt{X_{0}^{2}+Y_{0}^{2}+Z_{0}^{2}}}\right)=0 .
$$

But $a \neq h$, because if $a=h$ the $\Omega=\mathrm{S}$, which is a contradiction. Therefore $\frac{c \sqrt{2}}{2}(a-h) \neq 0$. Thus: $1-\frac{\sqrt{X^{2}+Y^{2}+Z^{2}}}{\sqrt{X_{0}^{2}+Y_{0}^{2}+Z_{0}^{2}}}=0$ and then

$$
\sqrt{X^{2}+Y^{2}+Z^{2}}=\sqrt{X_{0}^{2}+Y_{0}^{2}+Z_{0}^{2}} .
$$

b) $\boldsymbol{f}_{\mathbf{1}}$ is surjective.

Let $M^{\prime}\left(X^{\prime} ; Y^{\prime} ; Z^{\prime}\right) \in \mathrm{S}_{S A D}$ and $M(X ; Y ; Z)=\left[\Omega M^{\prime}\right) \cap \mathrm{P}_{S A D}$.

We have $\Omega M \wedge \Omega M^{\prime}=\overrightarrow{0}$

Because $\Omega, M$ and $M$ are collinear.

Thus,

$$
\left(\begin{array}{l}
X \\
Y \\
Z
\end{array}\right) \wedge\left(\begin{array}{l}
X^{\prime} \\
Y^{\prime} \\
Z^{\prime}
\end{array}\right)=\overrightarrow{0} \leftrightarrow\left\{\begin{array} { l } 
{ Y Z ^ { \prime } - Z Y ^ { \prime } = 0 } \\
{ - X Z ^ { \prime } + Z X ^ { \prime } = 0 } \\
{ X Y ^ { \prime } - Y X ^ { \prime } = 0 }
\end{array} \leftrightarrow \left\{\begin{array}{l}
X=\frac{X^{\prime}}{Z^{\prime}} Z \\
Y=\frac{Y^{\prime}}{Z^{\prime}} Z
\end{array} .\right.\right.
$$

Replacing expression of $X$ and $Y$ in equation of $\mathrm{P}_{S A D}$, we obtain:

$$
\frac{Y^{\prime}-X^{\prime}}{Z^{\prime}} Z=\frac{\sqrt{2}\left(h \sin \theta+\frac{c}{2} \cos \theta\right)}{h \cos \theta-\frac{c}{2} \sin \theta} Z+\frac{c \sqrt{2}(a-h)}{2 h \cos \theta-\operatorname{csin} \theta} .
$$

i.e

$Z\left(\frac{Y^{\prime}-X^{\prime}}{Z^{\prime}}-\frac{\sqrt{2}(2 h \sin \theta+\cos \theta)}{2 h \cos \theta-\operatorname{csin} \theta}\right)=\frac{c \sqrt{2}(a-h)}{2 h \cos \theta-\operatorname{csin} \theta}$.

i.e;

$$
Z=\frac{c \sqrt{2}(a-h) Z^{\prime}}{(2 h \cos \theta-c \sin \theta)\left(Y^{\prime}-X^{\prime}\right)-\sqrt{2}(2 h \sin \theta+\cos \theta) Z^{\prime}}
$$

Thus

$$
\begin{aligned}
& X=\frac{X^{\prime}}{Z^{\prime}} Z \\
& =\frac{c \sqrt{2}(a-h) X^{\prime}}{(2 h \cos \theta-\operatorname{csin} \theta)\left(Y^{\prime}-X^{\prime}\right)-\sqrt{2}(2 h \sin \theta+\cos \theta) Z^{\prime}} .
\end{aligned}
$$

Likewise:

$$
Y=\frac{Y^{\prime}}{Z^{\prime}} Z
$$

Then

$Y=\frac{Y^{\prime}}{Z^{\prime}} Z$

$$
=\frac{c \sqrt{2}(a-h) Y^{\prime}}{(2 h \cos \theta-\operatorname{csin} \theta)\left(Y^{\prime}-X^{\prime}\right)-\sqrt{2}(2 h \sin \theta+\cos \theta) Z^{\prime}} .
$$


To conclude that $f_{1}$ is surjective, we will show that $f_{1}(M)=M^{\prime} . M \in \Delta_{S A D}$.

$\mathrm{P}_{S \Omega A}, \mathrm{P}_{S \Omega D}$ and $\mathrm{P}_{D \Omega A}$ are bounds plans of regions $\mathrm{S}_{S A D}$ and $\Delta_{S A D}$ therefore, for all $M^{\prime} \in \mathrm{S}_{S A D},\left(\Omega M^{\prime}\right) \cap \Delta_{S A D}$ is one point set. It follow bay construction that $M \in \Delta_{S A D}$.

Denote:

$Q_{M^{\prime}}=\frac{c \sqrt{2}(a-h)}{(2 h \cos \theta-\sin \theta)\left(Y^{\prime}-X^{\prime}\right)-\sqrt{2}(2 h \sin \theta+\cos \theta) Z^{\prime}}$.

We shall prove that $f_{1}(M)=M^{\prime}$. We have

$f_{1}(M)=\left(\frac{R X}{\sqrt{X^{2}+Y^{2}+Z^{2}}}, \frac{R Y}{\sqrt{X^{2}+Y^{2}+Z^{2}}}, \frac{R Z}{\sqrt{X^{2}+Y^{2}+Z^{2}}}\right)$.

But

$$
\begin{aligned}
& \frac{R X}{\sqrt{X^{2}+Y^{2}+Z^{2}}}=\frac{R Q_{M^{\prime}} X^{\prime}}{\sqrt{Q_{M^{\prime}}^{2} X^{\prime 2}+Q_{M^{\prime}}^{2} Y^{\prime 2}+Q_{M}^{2},^{\prime 2}}} \\
& =\frac{R Q_{M^{\prime}} X^{\prime}}{\left|Q_{M^{\prime}}\right| \sqrt{X^{\prime 2}+Y^{\prime 2}+Z^{\prime 2}}} \\
& =\frac{R X^{\prime}}{\sqrt{X^{\prime 2}+Y^{\prime 2}+Z^{\prime 2}}} \text { because } Q_{M^{\prime}}>0 \\
& =X^{\prime} \text { because } \sqrt{X^{\prime 2}+Y^{\prime 2}+Z^{\prime 2}}=R
\end{aligned}
$$

since $M^{\prime} \in \mathrm{S}_{S A D}$

In the same manner, we prove that

$$
\frac{R Y}{\sqrt{X^{2}+Y^{2}+Z^{2}}}=Y^{\prime}
$$

And $\frac{R Z}{\sqrt{X^{2}+Y^{2}+Z^{2}}}=Z^{\prime}$.

i.e; $f_{1}(M)=M^{\prime}$; then $f_{1}$ is surjective.

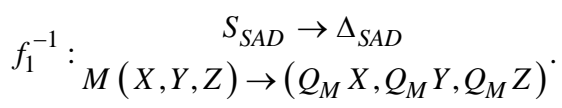

We shall now prove that $f_{1}$ is bi-continuous.

\section{c) $f_{1}$ is continuous.}

Let $M_{0}\left(X_{0} ; Y_{0} ; Z_{0}\right) \in \Delta_{S A D}$, and $\varepsilon>0$, we shall fine $\gamma_{\varepsilon}$ such that

$$
\left\|M-M_{0}\right\| \leq \gamma_{\varepsilon} \Rightarrow\left\|f_{1}(M)-f_{1}\left(M_{0}\right)\right\| \leq \varepsilon .
$$

We have:

$$
\begin{aligned}
& f 1(M)-f 1(M 0) \\
& =\operatorname{R}\left(\begin{array}{l}
\frac{X}{\sqrt{X^{2}+Y^{2}+Z^{2}}}-\frac{X_{0}}{\sqrt{X_{0}^{2}+Y_{0}^{2}+Z_{0}^{2}}} \\
\frac{Y}{\sqrt{X^{2}+Y^{2}+Z^{2}}}-\frac{Y_{0}}{\sqrt{X_{0}^{2}+Y_{0}^{2}+Z_{0}^{2}}} \\
\frac{Z}{\sqrt{X^{2}+Y^{2}+Z^{2}}}-\frac{Z_{0}}{\sqrt{X_{0}^{2}+Y_{0}^{2}+Z_{0}^{2}}}
\end{array}\right)=R\left(\begin{array}{l}
\frac{X}{\|\overrightarrow{\Omega M}\|}-\frac{X_{0}}{\left\|\overrightarrow{\Omega M_{0}}\right\|} \\
\frac{Y}{\|\overrightarrow{\Omega M}\|}-\frac{Y_{0}}{\| \overrightarrow{\Omega M_{0} \|}} \\
\frac{X}{\|\overrightarrow{\Omega M}\|}-\frac{Z_{0}}{\left\|\overrightarrow{\Omega M_{0}}\right\|}
\end{array}\right) .
\end{aligned}
$$

Thus, denoting:

$$
k=\frac{R}{\|\overrightarrow{\Omega M}\|\left\|\overrightarrow{\Omega M}_{0}\right\|} ; k_{M}=\|\overrightarrow{\Omega M}\| ;
$$

$k_{M_{0}}=\left\|\overrightarrow{\Omega M_{0}}\right\|$ we obtain:

$$
\left\|f_{1}(M)-f_{1}\left(M_{0}\right)\right\|=k \sqrt{\left[\begin{array}{l}
\left(k_{M_{0}} X-k_{M} X_{0}\right)^{2} \\
+\left(k_{M_{0}} Y-k_{M} Y_{0}\right)^{2} \\
+\left(k_{M_{0}} Z-k_{M} Z_{0}\right)^{2}
\end{array}\right]} .
$$

But in R', $\forall M \in \Delta_{S A D}$, we have: $Z>0$. Since $k_{M}, k_{M_{0}} \in$ IR, then one is bigger than another. Without change the general case, suppose that $k_{M_{0}} \leq k_{M}$, then $\frac{k_{M_{0}}}{k_{M}} \leq 1$.

Therefore, $\frac{k_{M_{0}}}{k_{M}}\left\|X-X_{0}\right\| \leq\left\|X-X_{0}\right\|$;

$$
\frac{k_{M_{0}}}{k_{M}}\left\|Y-Y_{0}\right\| \leq\left\|Y-Y_{0}\right\|
$$

and

$$
\frac{k_{M_{0}}}{k_{M}}\left\|Z-Z_{0}\right\| \leq\left\|Z-Z_{0}\right\|
$$

thus,

$$
\begin{aligned}
& \left\|f_{1}(M)-f_{1}\left(M_{0}\right)\right\| \\
& =\frac{R}{\left\|\overrightarrow{\Omega M_{0}}\right\|} \sqrt{\left(X-X_{0}\right)^{2}+\left(Y-Y_{0}\right)^{2}+\left(Z-Z_{0}\right)^{2}} \\
& =\frac{R}{\left\|\overrightarrow{\Omega M_{0}}\right\|}\left\|\overrightarrow{M_{0} M}\right\| .
\end{aligned}
$$

Consequently:

$$
\left\|\overrightarrow{M_{0} M}\right\| \leq \frac{\varepsilon\left\|\overrightarrow{\Omega M_{0}}\right\|}{R} \rightarrow\left\|f_{1}(M)-f_{1}\left(M_{0}\right)\right\| \leq \varepsilon .
$$

It we be enough to take $\gamma \varepsilon=\frac{\varepsilon\left\|\overrightarrow{\Omega M_{0}}\right\|}{R}$ and then $f_{1}$ is continuous.

d) Let us prove that $\boldsymbol{f}_{\mathbf{1}}^{-1}$ is continuous.

Let $M_{0}\left(X_{0} ; Y_{0} ; Z_{0}\right) \in \mathrm{S}_{S A D}$, and $\varepsilon>0$, we shall fine $\gamma_{\varepsilon>0}$ such that $\left\|M-M_{0}\right\| \leq \gamma_{\varepsilon} \Rightarrow\left\|\boldsymbol{f}_{\mathbf{1}}^{-\mathbf{1}}(M)-\boldsymbol{f}_{\mathbf{1}}^{-\mathbf{1}}\left(M_{0}\right)\right\| \leq \varepsilon$.

We have

$$
\begin{aligned}
& \left\|f_{1}^{-1}(\mathrm{M})-f_{1}^{-1}\left(M_{0}\right)\right\| \\
& =\left\|\left(Q X-Q_{0} X_{0}, Q Y-Q_{0} Y_{0}, Q Z-Q_{0} Z_{0}\right)\right\| \\
& =\sqrt{\left(Q X-Q_{0} X_{0}\right)^{2}+\left(Q Y-Q_{0} Y_{0}\right)^{2}+\left(Q Z-Q_{0} Z_{0}\right)^{2}} \\
& =Q_{0} \sqrt{\left(\frac{Q}{Q_{0}} X-X_{0}\right)^{2}+\left(\frac{Q}{Q_{0}} Y-Y_{0}\right)^{2}+\left(\frac{Q}{Q_{0}} Z-Z_{0}\right)^{2}} .
\end{aligned}
$$


Since $Q, Q_{0} \in \mathrm{R}$, then one of them is bigger than other. Without change the generality

Suppose that $Q \leq Q_{0}$, thus $\frac{Q}{Q_{0}} \leq 1$.

Since $Z>0$, then

$$
\begin{aligned}
& \frac{Q}{Q_{0}}\left\|X-X_{0}\right\| \leq\left\|X-X_{0}\right\| ; \\
& \frac{Q}{Q_{0}}\left\|Y-Y_{0}\right\| \leq\left\|Y-Y_{0}\right\| \\
& \text { and } \frac{Q}{Q_{0}}\left\|Z-Z_{0}\right\| \leq\left\|Z-Z_{0}\right\| .
\end{aligned}
$$

Therefore

$$
\begin{aligned}
& \left\|f_{1}^{-1}(\mathrm{M})-f_{1}^{-1}\left(M_{0}\right)\right\| \\
& =Q_{0} \sqrt{\left(X-X_{0}\right)^{2}+\left(Y-Y_{0}\right)^{2}+\left(Z-Z_{0}\right)^{2}} \\
& =Q_{0}\left\|\overrightarrow{M_{0} M}\right\| .
\end{aligned}
$$

It is enough to take $\gamma \varepsilon=\frac{\varepsilon}{Q_{0}}$. From $a$ ), $b$ ), $c$ ) and $d$ ) we conclude that $f_{1}$ is an homeomorphism from $\Delta_{S A D}$ to $\mathrm{S}_{S A D}$. Remark 3.1. Using the same methods, we prove that $f_{2}, f_{3}$ and $f_{4}$ which transform faces $\Delta_{S A B}, \Delta_{S B C}$ and $\Delta_{S C A}$ of Euclidean pyramid to their spherical counterpart $\mathrm{S}_{S A B}, \mathrm{~S}_{S B C}$ and $\mathrm{S}_{S C A}$ respectively.

\subsection{On Homeomorphism between Euclidean Pyramid and Is Spherical Counterpart}

\section{Theorem 3.1.}

The map f: $I R^{3} \rightarrow I R^{3}$

$$
M \rightarrow\left\{\begin{array}{c}
f_{i}(M) \text { if } M \in \Delta_{i}, i \in\{1,2,3,4\} \\
f_{i}^{-1}(M) \text { if } M \epsilon S_{i}, i \varepsilon\{1,2,3,4\} \\
M \text { if not }
\end{array}\right.
$$

is an homeomorphism transforming the Euclidean pyramid $S A B C D$ to is spherical counterpart.

Proof: We shall prove that $f$ is bijective, continuous with its inverse.

\section{a) Let us prove that $f$ is injective.}

Let $M, M^{\prime} \in \mathrm{IR}^{3}$ such that $f(M)=f\left(M^{\prime}\right)$. We wish to prove that $M=M^{\prime}$.

- If there is $i \in\{1,2,3,4\}$ such that $M \in \Delta_{i}$, then $f(M)=$ $f_{i}(M)=f\left(M^{\prime}\right)$. Without change the generality, suppose that $i=1$. Let us prove that necessarily, $M^{\prime} \in \Delta_{1}$. Suppose that $M^{\prime} \notin \Delta_{1}$. Then, either $\exists j \in\{1,2,3,4\}$ such that $M^{\prime} \in S_{j}$ $\backslash\{S, A, D\}$, or $\exists j \in\{2,3,4\}$ such that $M^{\prime} \in \Delta_{j} \backslash\left\{\Delta_{1}\right\}$, or $M^{\prime} \notin \Delta_{j}$ $\cup \mathrm{S}_{j} \forall j \in\{1,2,3,4\}$.

- If $M^{\prime} \in \mathrm{S}_{j} \backslash\{S, A, D\}$, then $f\left(M^{\prime}\right)=f_{j}^{-1}\left(M^{\prime}\right)=f_{1}(M)$, this is absurd because $f_{1}(M) \in \mathrm{S}_{S A D}$ and $f_{j}^{-1}\left(M^{\prime}\right) \in \Delta_{j} \backslash\{S, A, D\}$. However, equality hold only for points $S, A$ and $D$; because:

$$
\begin{gathered}
S_{S A D \cap \Delta_{j}}=\{S, A, D\} \text { if } j=1 \\
S_{S A D \cap \Delta_{j}}=\{S, A\} \text { if } j=2
\end{gathered}
$$

$$
\begin{gathered}
S_{S A D \cap} \Delta_{j}=\{S, D\} \text { if } j=3 \\
S_{S A D} \triangle \Delta_{j}=\{S\} \text { if } j=4 .
\end{gathered}
$$

Thus, $\mathrm{S}_{S A D} \cap \Delta_{j} \backslash\{S, A, D\}=\emptyset \forall j$.

- If $M^{\prime} \in \Delta_{j} \backslash\left\{\Delta_{1}\right\}$, then $f\left(M^{\prime}\right)=f_{j}\left(M^{\prime}\right), \forall j \in\{2,3,4\}$. Thus, $f_{1}(M)=f_{j}\left(M^{\prime}\right) \forall j \in\{2,3,4\}$.

This last equality is absurd; because; if not two points will be equal whenever there are on two disjoints spherical triangles, since $f_{1}(M) \in \mathrm{S}_{1}$ and $f_{j}\left(M^{\prime}\right) \in \mathrm{S}_{j} \backslash\left\{\mathrm{S}_{1}\right\}(j \neq 1)$.

- If $\forall j \in\{1,2,3,4\}, M^{\prime} \notin \Delta_{j} \cup \mathrm{S}_{j}$, then $f\left(M^{\prime}\right)=M^{\prime}=f_{1}(M)$, this is absurd because $f_{1}(M) \in \mathrm{S}_{1}$ and $M^{\prime} \notin \Delta_{j} \cup \mathrm{S}_{j}$, i.e; $M^{\prime} \notin$ $\mathrm{S}_{1}$

In the same manner, we prove that $i \in\{2,3,4\}$, if $M \in \Delta_{i}$, then it will be the same to $M^{\prime}$.

Thus, we will have $f_{i}(M)=f_{i}\left(M^{\prime}\right) \forall i \in\{1,2,3,4\}$ and then $M=M^{\prime}$ because $\forall i, f_{i}$ is an homeomorphism.

- $\forall i \in\{1,2,3,4\}$, using the above method, we prove that if we have $M \in \mathrm{S}_{i}$, then we will have necessarily $M^{\prime} \in \mathrm{S}_{i}$ and in this case we will have $f(M)=f_{i}^{-1}(M)=$ $f_{i}^{-1}\left(M^{\prime}\right)=f\left(M^{\prime}\right)$, and then $M=M^{\prime}$ because $\forall i, f_{i}^{-1}$ is an homeomorphism.

Therefore $f$ is injective.

- $\forall i \in\{1,2,3,4\}$, We also follow the above method to show that if we have $\mathrm{M} \notin \mathrm{S}_{i} \cup \Delta_{i}$, then we will have necessarily $M^{\prime} \notin \mathrm{S}_{i} \cup \Delta_{i}$ and in this case we will have $f(M)$ $=M=f\left(M^{\prime}\right)=M^{\prime}$, and then $f$ is injective.

b) Let us prove that $f$ is surjective.

Let $M^{\prime} \in \mathrm{IR}^{3}$, we shall fine $M \in \mathrm{IR}^{3}$ such that $f(M)=M^{\prime}$.

$\sqrt{ }$ If $\exists i \in\{1,2,3,4\}$ such that $M^{\prime} \in \Delta_{i}$, then $\{M\}=\mathrm{S} \cap$ $\left(\Omega M^{\prime}\right)$.

$\sqrt{ }$ If $\exists i \in\{1,2,3,4\}$ such that $M^{\prime} \in \mathrm{S}_{i},\{M\}=\mathrm{P}_{i} \cap$ $\left(\Omega M^{\prime}\right)$.

$\sqrt{ }$ If $\forall i \in\{1,2,3,4\} M^{\prime} \notin \mathrm{S}_{i} \cup \Delta_{i}, M=M^{\prime}$.

It follow from $(a)$ and $(b)$, that $f$ a bijection.

c) Let us prove that $\boldsymbol{f}$ is continuous.

Let $M_{0} \in \mathrm{IR}^{3}$ and $\varepsilon>0$, we seek $\gamma>0$ such that $\forall M \in \mathrm{IR}^{3},\left\|M-M_{0}\right\|<\gamma \Rightarrow\left\|f(M)-f\left(M_{0}\right)\right\|<\varepsilon$.

Since $\Delta_{1} \cup \Delta_{2} \cup \Delta_{3} \cup \Delta_{4}$ and $S_{1} \cup S_{2} \cup S_{3} \cup S_{4}$ are continuous surfaces and $\forall i, j \in\{1,2,3,4\}, \Delta_{i} \cap \Delta_{j}, \Delta_{i} \cap S_{j}$, $S_{i} \cap S_{j}$ are non-empty set, since $S$ belong to them. Then it exist $(k, p) \in \mathrm{IN}^{*},(I, J) \in \mathrm{P}\left(\mathrm{N}^{*}\right) \times \mathrm{P}\left(\mathrm{N}^{*}\right)$, and $(S, T, U, V) \in$ $\left(\mathrm{P}\left(\mathrm{IR}^{3}\right)\right)^{4}$, where $I=\left\{i \in \mathrm{IN}^{*} / 1 \leq i \leq k\right\}, J=\left\{j \in \mathrm{IN}^{*} / k+\right.$ $1 \leq j \leq k+p\}, S=\left\{M_{i} \in \Delta_{i}, i \in I\right\}, T=\left\{M_{j} \in \Delta_{j}, j \in J\right\}, U$ $=\left\{M_{i} \in \mathrm{S}_{i}, i \in I\right\}$ and $V=\left\{M_{j} \in \mathrm{S}_{j}, j \in J\right\}$; such that $\forall M \in$ $I R^{3}$, there is a sequence of points of $S$, a sequence of $T$, a sequence of points of $U$, and a sequence of points of $V$, such that $\left\|M_{i}-M_{i+1}\right\| \leq\left\|M-M_{0}\right\|$ with $i<k-1 ; i>k ;\left\|M_{k}-S\right\| \leq\left\|M-M_{0}\right\|$ and $\left\|M_{k+1}-S\right\| \leq$ $\left\|M-M_{0}\right\|$.

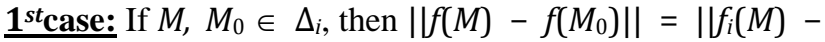
$f_{i}\left(M_{0}\right) \|$. As $f_{i}$ is continuous on $\Delta_{i}$, then for $\varepsilon>0, \exists \alpha>0$ such that $\left\|M-M_{0}\right\|<\alpha \Rightarrow\left\|f_{i}(M)-f_{i}\left(M_{0}\right)\right\|<\varepsilon$. It will be enough to take $\gamma=\alpha$ and the result is proved.

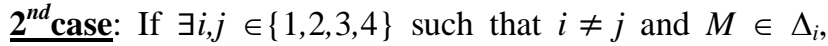
$M_{0} \in \Delta_{j}$, then there is $\left(M_{t}\right) \subset S$ and $\left(K_{t}\right) \subset T$ such that: $\left\|M_{i}-M_{i+1}\right\| \leq\left\|M-M_{0}\right\| ;\left\|M_{k}-S\right\| \leq\left\|M-M_{0}\right\| ;\left\|S-K_{k+1}\right\| \leq$ $\left\|M-M_{0}\right\| ;\left\|K_{i}-K_{i+1}\right\| \leq\left\|M-M_{0}\right\|$. But 


$$
\begin{aligned}
& \left\|f(M)-f\left(M_{0}\right)\right\| \leq\left\|f(M)-f\left(M_{1}\right)\right\| \\
& +\sum_{i=1}^{k-1}\left\|f\left(M_{i}\right)-f\left(M_{i+1}\right)\right\|+\left\|f\left(M_{k}\right)-f(S)\right\| \\
& +\left\|f(S)-f\left(M_{k+1}\right)\right\|+\sum_{i=k+1}^{k+p-1}\left\|f\left(M_{i}\right)-f\left(M_{i+1}\right)\right\| \\
& =\left\|f_{i}(M)-f_{i}\left(M_{1}\right)\right\|+\sum_{t=1}^{k-1}\left\|f_{i}\left(M_{t}\right)-f_{i}\left(M_{t+1}\right)\right\| \\
& +\left\|f_{i}\left(M_{k}\right)-f_{i}(S)\right\|+\left\|f_{j}(S)-f_{j}\left(M_{k+1}\right)\right\| \\
& +\sum_{t=k+1}^{k+p-1}\left\|f_{j}\left(M_{t}\right)-f_{j}\left(M_{t+1}\right)\right\| .
\end{aligned}
$$

As $f_{i}$ and $f_{j}$ are continuous, then, for

$$
\frac{\varepsilon}{k+p+1}>0
$$

it exist $\left(\alpha_{i}\right) \in I R_{+}^{*}$ such that

$$
\begin{gathered}
\left\|M-M_{1}\right\|\left\langle\alpha_{1} \rightarrow \| f_{i}(M)-f_{i}\left(M_{1}\right)\right|<\frac{\varepsilon}{k+p+1} \\
\left\|M_{t}-M_{t+1}\right\|<\alpha_{t} \rightarrow\left\|f_{i}\left(M_{t}\right)-f_{i}\left(M_{t+1}\right)\right\| \\
<\frac{\varepsilon}{k+p+1} t \epsilon[2 \ldots k] \\
\left\|M_{k}-S\right\|<\alpha_{k+1} \rightarrow\left\|f_{i}\left(M_{k}\right)-f_{i}(S)\right\|<\frac{\varepsilon}{k+p+1} \\
\left\|S-M_{k+1}\right\|<\alpha_{k+2} \rightarrow\left\|f_{j}(S)-f_{j}\left(M_{k+1}\right)\right\|<\frac{\varepsilon}{k+p+1} \\
\left\|M_{t}-M_{t+1}\right\|<\alpha_{t} \rightarrow\left\|f_{j}\left(M_{t}\right)-f_{j}\left(M_{t+1}\right)\right\| \\
<\frac{\varepsilon}{k+p+1} t \epsilon[k+1 \ldots k+p+1]
\end{gathered}
$$

Taking $\gamma=\min \left\{\alpha_{i}, i \in[1 \ldots k+p+1]\right\}$, then $\left\|M-M_{0}\right\|<$ $\gamma \Rightarrow\left\|f(M)-f\left(M_{0}\right)\right\|<\varepsilon$.

In the same manner, we prove the other cases.

c) Continuity of $\boldsymbol{f}^{-1}$.

$$
\begin{gathered}
f^{-1}: I R^{3} \rightarrow I R^{3} \\
M \rightarrow\left\{\begin{array}{c}
f_{i}^{-1}(M) \text { if } M \in \Delta_{i}, i \in\{1,2,3,4\} \\
f_{i}(M) \text { if } M \in S_{i}, i \varepsilon\{1,2,3,4\} \\
M \text { if not }
\end{array}\right.
\end{gathered}
$$

The continuity of $f^{-1}$ is proving as that of $f$.

\section{References}

[1] Anderson, J. W. (2006). Hyperbolic geometry. Springer Science \& Business Media.

[2] Dongho, J.\& Kemmegne Fopossi, S. (2019) Homologue Spérique d'une pyramide Euclidienne. EUE, ISBN 978-613-845473-1.

[3] Fresnel, J. (1996). Méthodes modernes en géométrie (Vol. 2010). Paris: Hermann.

[4] Frenkel, J. (1977). Géométrie pour l'élève-professeur: Actualités scientifiques et industrielles. Hermann.

[5] Fitzpatrick, R. (2008). Euclid's elements of geometry. (pp. 195222).

[6] Guinot, M. (2009). Arithmétique pour amateurs: (par un autodidacte). 3." Ce diable d'homme" D'Euler. Aléas.

[7] Lebossé, C., \& Hémery, C. (1997). Géométrie: classe de mathématiques: programmes de 1945. Jacques Gabay.

[8] Lebossé, C., \& Hémery, C. (1962). Arithmétique, algèbre, et géométrie: classe de quatrième. F. Nathan.

[9] Lebossé, C., \& Hémery, C. (1949). Arithmétique et dessin géométrique: classe de 6e des lycées, des collèges et des cours complémentaires: programme de 1947. F. Nathan.

[10] Legendre, A. M. (1853). Eléments de géométrie (Vol. 1). Comité de liquidation.

[11] Lelong-Ferrand, J. (1985). Les fondements de la géométrie. Presses Universitaires de France-PUF.

[12] Serret, J. A. (1868). Traité de trigonométrie.

[13] Sortais, Y., \& Sortais, R. (1997). La géométrie du triangle. Hermann32.

[14] Troyanov, M. (2009). Cours de géométrie. Presses polytechniques et universitaires romandes. 